\title{
Photogrammetric Mobile Satellite Service Prediction
}

\author{
Riza Akturan \\ Wolfhard J. Vogel \\ Electrical Engineering Research Laboratory \\ The University of Texas at Austin
}

\begin{abstract}
Photographic images of the sky were taken with a camera through a fisheye lens with a $180^{\circ}$ field-of-view. The images of rural, suburban, and urban scenes were analyzed on a computer to derive quantitative information about the elevation angles at which the sky becomes visible. Such knowledge is needed by designers of mobile and personal satellite communications systems and is desired by customers of these systems. The 90th percentile elevation angle of the skyline was found to be $10^{\circ}, 17^{\circ}$, and $51^{\circ}$ in the three environments. At $8^{\circ}, 75 \%, 75 \%$, and $35 \%$ of the sky was visible, respectively. The elevation autocorrelation fell to zero with a $72^{\circ} \mathrm{lag}$ in the rural and urban environment and a $40^{\circ} \mathrm{lag}$ in the suburb. Mean estimation errors are below $4^{\circ}$.
\end{abstract}

\section{INTRODUCTION}

All economically feasible mobile satellite communications systems, whether targeted for vehicular or personal use, and whether operating at UHF-, L-, S-Band frequencies or higher, are ultimately performance limited by the three non-exclusive propagation states in which the mobile earth station (MES) can find itself. The MES can either be

1. shadowed by trees,

2. blocked by mountains and structures such as buildings and overpasses,

3. or receiving multipath echoes generated by reflectors such as terrain and buildings or scatterers such as trees, utility poles and building edges.

Many measurement campaigns have been carried out in the US and abroad [1] to establish a database for system simulation as well as for statistical and analytical performance prediction. At this time, the significant environmental parameters and their electromagnetic repercussions are reasonably well understood. However, efforts of generalized modeling of the effects engendered by these three states and of producing reliable system performance predictions are hampered by the requirement that one needs to possess knowledge of the environment near the MES at a large number of possible locations. Obtaining such knowledge by carrying out systematic measurement campaigns using real or simulated satellite transmissions is very timeconsuming and expensive. A more convenient method of determining the incidence of the three fade states is needed.

Previous measurements of optical brightness along the line-of-sight to a balloon-borne transmitter have demonstrated that there exists a statistical linkage between fading and optical intensity [2]. We expand on that point measurement by developing a system that evaluates full images of the upper half-sphere and determines where the sky is clear, where it is shadowed by vegetation, and where it is blocked. Assuming that enough images are acquired in a specified environment, one can develop statistics for the three fade states as a function of elevation and azimuth angles and these can later be combined with statedependent propagation data to produce realistic simulated data or attenuation statistics. The image data can then be used, for instance, to predict fading probability as a function of elevation angle or to forecast the diversity gain of a satellite system employing satellite diversity, all in a chosen environment.

The ultimate goal of this research is to develop automatic image-recognition 
techniques that can be employed to derive personal and mobile satellite propagation predictions from video images taken with a fish-eye lens. If fully successful, then images will be analyzed in real time at the 30 frames/second rate to identify each fade state as a function of azimuth and elevation. Our near-term goal was more modest. We acquired and analyzed images taken through a fish-eye lens with a $35 \mathrm{~mm}$ still-camera in a rural, a suburban, and two urban locales. Instead of the ternary classification, we restricted the recognition to just two states: clear sky or not.

In the following, we present a brief system description, some information about the detection algorithms, and the statistical results from the initial batch of images. We will also outline the future direction of our efforts and offer some conclusions.

\section{SYSTEM DESCRIPTION}

The images described and evaluated here were acquired with a $35 \mathrm{~mm}$ camera and a fisheye lens with a full $180^{\circ}$ field of view. The camera was always mounted on a leveled tripod and a compass was used to align the top of each frame towards north. The photos were taken on slide film and the slides were scanned into a personal computer. The fisheye lens has also been coupled to a video camera in a system which can be mounted to the roof of a car and includes a GPS receiver, which provides location, vehicle speed and heading. At a later time, the video recordings will be played into a frame-grabber for analysis.

\section{SOFTWARE ALGORITHMS}

The current binary image-identification software starts by reducing the color image to a 256-level gray-scale and then unwrapping it, i.e., converting from a circular image in which the zenith direction is at the center to a rectangular coordinate system in which zenith is at the top of the frame. The second derivative of the histogram of the image, after filtering, is then used to estimate the graylevel threshold value separating sky and objects [3]. After this procedure, a binary image can be produced from which all the results presented here are derived. These are, for each image, (1) the elevation angle of the sky-line as a function of azimuth [4] and (2) the fraction of visible sky as a function of elevation. As a visual aid to interactively assess the validity of the calculations, a contour line representing the first result can be drawn over the rectangular picture.

To derive ternary instead of just binary object identification, more processing will be required and is now under development. This includes object locating, and object identification, edge-detection, low-pass filtering, derivatives and statistical algorithms. Artificial intelligence procedures [5] will be used for classifying the objects into three separate groups. Pre-defined templates [6] have been avoided for the sake of faster execution. The result of these additional procedures will be a clear-shadowed-blocked image description, which will allow us to calculate the necessary statistics for a given image.

\section{INITIAL RESULTS}

Four environments in Central Texas were sampled, rural, suburban, and two cities (Austin and San Antonio). In the rural case, a total of 43 images were acquired along a sparsely tree-lined rural route connecting a small city to Austin, about one picture per mile on the side of the road. In the suburban case, 90 images were taken in a neighborhood with mostly single-story houses and relatively young trees, which did not form canopies over the road. In the central business districts (CBD) of Austin and San Antonio, 119 and 105 images were taken, respectively. For the last three cases, pictures were taken mid-block and at block corners with the camera at the street-side of the sidewalk. 
After determining the skyline for each image (see the example in Fig. 1), i.e., the highest elevation angle separating sky and terrestrial objects at each azimuth, statistics of skyline angles were calculated and averaged over all images in a particular environment. As expected, the average elevation angle of the skyline at each location strongly depends on the environment. The mean, 90th percentile and maximum elevation for the rural, suburban and Austin CBD environs are shown in Figs. 2 to 4 .

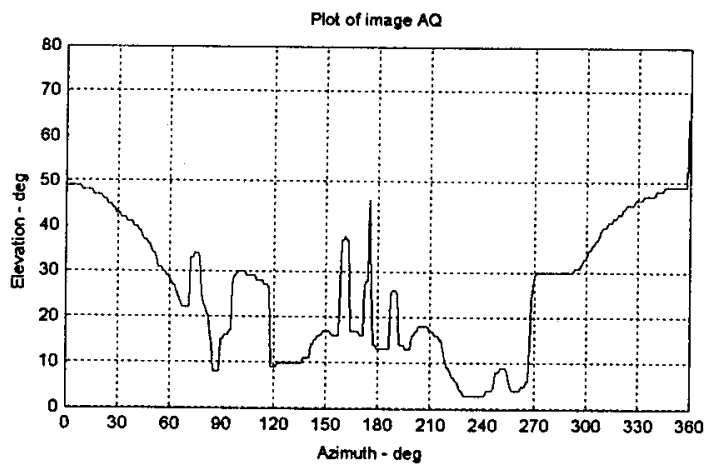

Fig. 1 Example of a skyline image (SA)

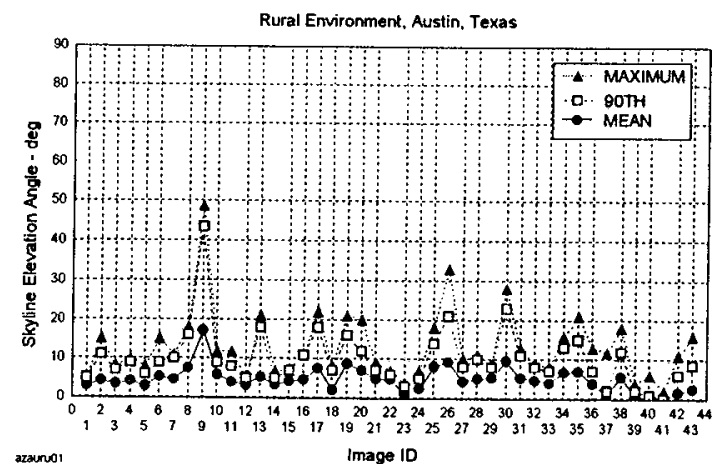

Fig. 2 Rural image statistics

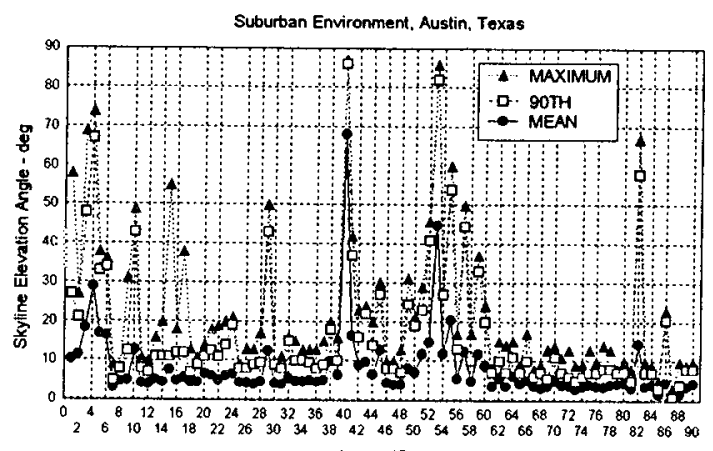

Fig. 3 Suburban skyline statistics

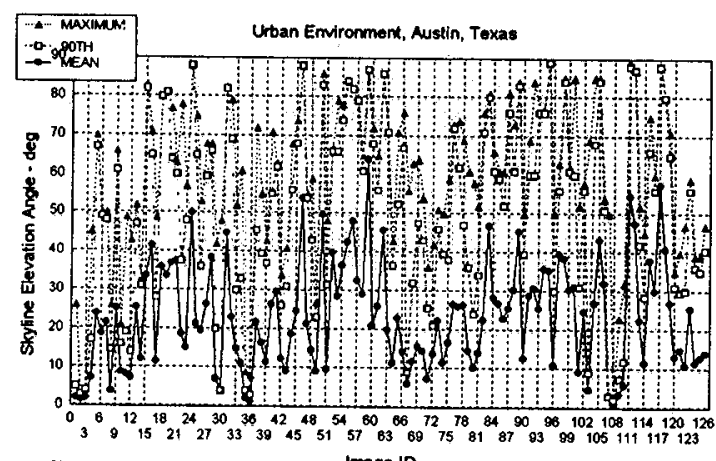

Fig. 4 Austin urban skyline statistics

After averaging over all images in a specific environment, the overall statistics in Table 1 are found.

Table 1: Average Skyline Statistics

\begin{tabular}{lccc} 
Environment & Mean $\left(^{\circ}\right.$ & STD $\left(^{\circ}\right)$ & $90 \%\left(^{\circ}\right)$ \\
\hline rural & 4.8 & 3.5 & 10.0 \\
suburban & 7.9 & 5.9 & 16.9 \\
Austin CBD & 23.5 & 18.8 & 50.5 \\
San Ant. CBD & 26.4 & 18.1 & 52.6
\end{tabular}

The 90th percentile of the skyline elevation angle follows a normal distribution, except in the suburban case.

The percentage of sky visible at a given elevation angle has been plotted in Figs. 5 and 6 for the Austin rural and urban environments. In the former, at $8^{\circ}$ elevation over $75 \%$ of the sky are visible (median), in the latter, only $35 \%$ of the sky can bee seen.

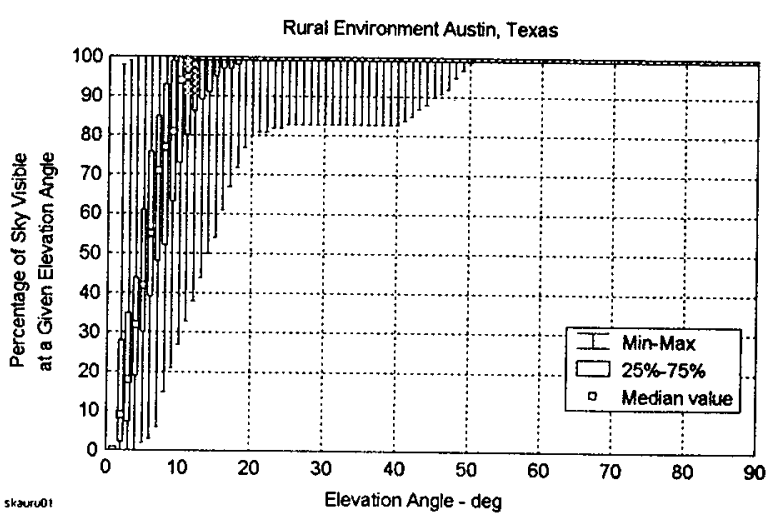

Fig. 5 Percent sky visible in rural Austin 


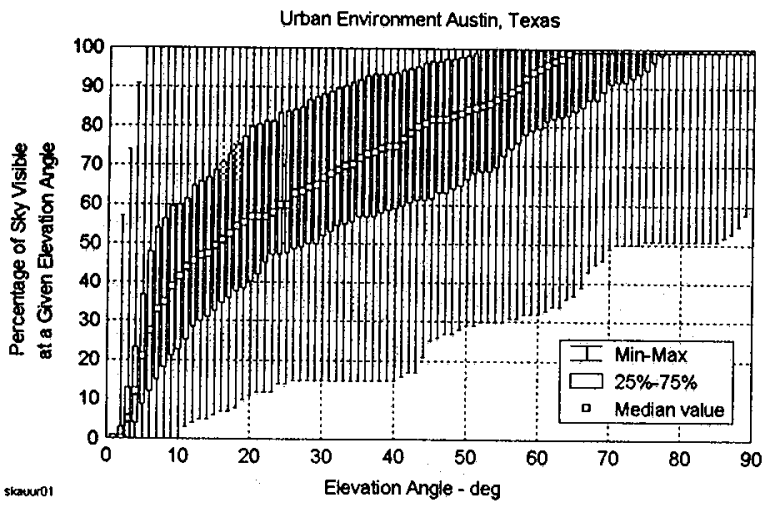

Fig. 6 Percent sky visible in urban Austin

The elevation angle distributions of the skylines for selected images (all with nearly environment-average mean elevation) are depicted in Fig. 7. The lognormal distribution has been fit to each histogram, but the fit is not very close.
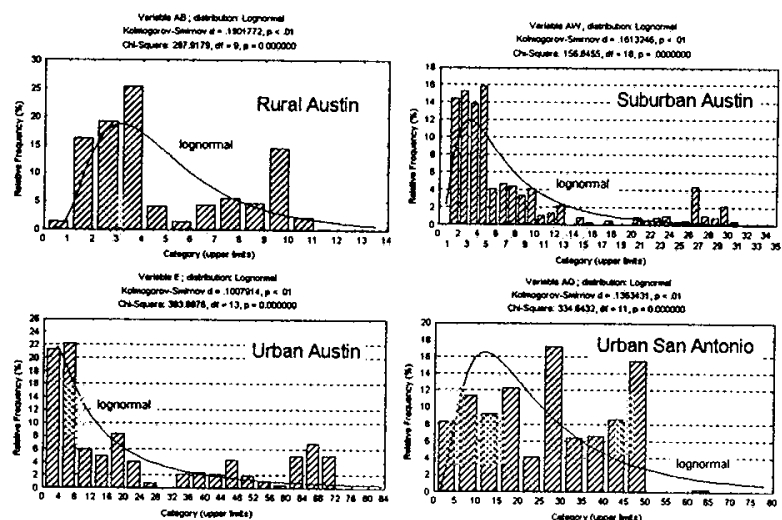

Fig. 7 Skyline elevation histograms

The autocorrelation of the skyline elevation vs. azimuth angle for the above selected images falls off linearly to 0 at a lag of near $70^{\circ}$, except for the suburban environment, where it reduces to 0 at $40^{\circ}$ lag. Figs. 8 and 9 show the autocorrelation for the suburban and urban Austin environs. The skyline image is also inserted into these graphs.

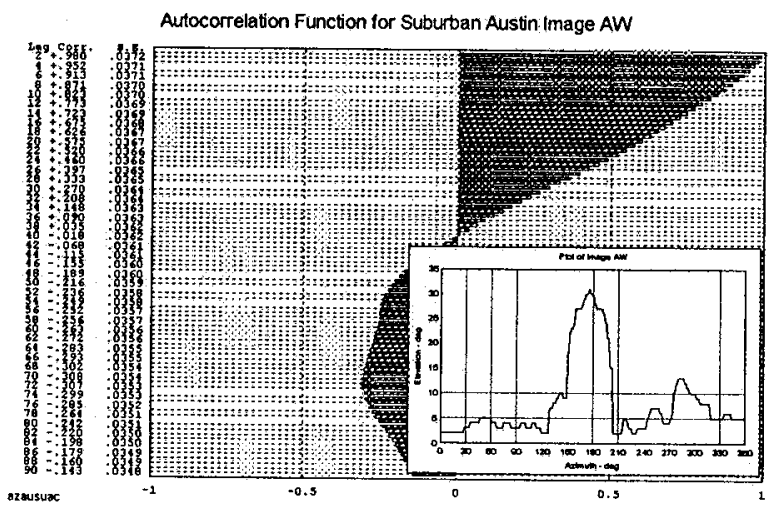

Fig. 8 Suburban skyline autocorrelation

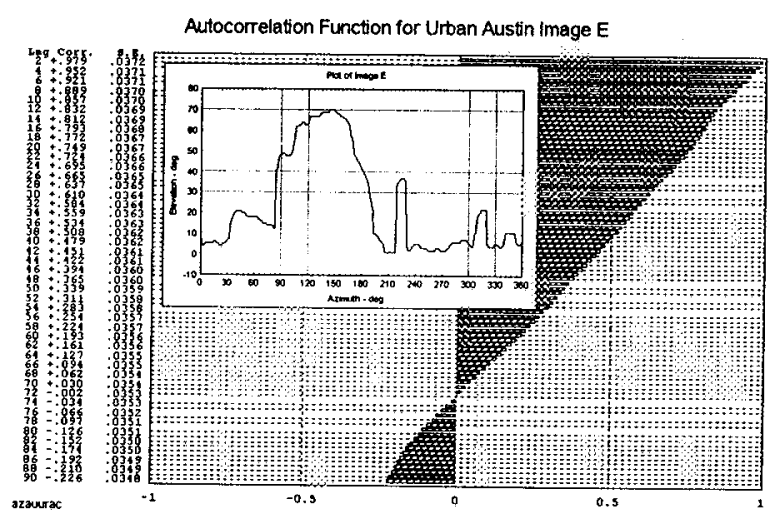

Fig. 9 Urban skyline autocorrelation

Errors in recognizing the correct skyline elevation angles were quantified by comparing the elevations found by the program with those derived by manually tracing selected images with a mouse. The difference, although to some degree dependent on the skill of the mouse operator, is taken as the error of the image recognition program. Fig. 10 summarizes the errors on an image-by-image basis, Fig. 11 depicts an overall box plot of the error for the four environments. The largest error, with a mean of $4^{\circ}$ and a standard deviation of about $3^{\circ}$, arose in the suburban environment. As this was the only environment with significant numbers of trees, the error can be explained by the uncertainty in defining the tree-sky boundary. 

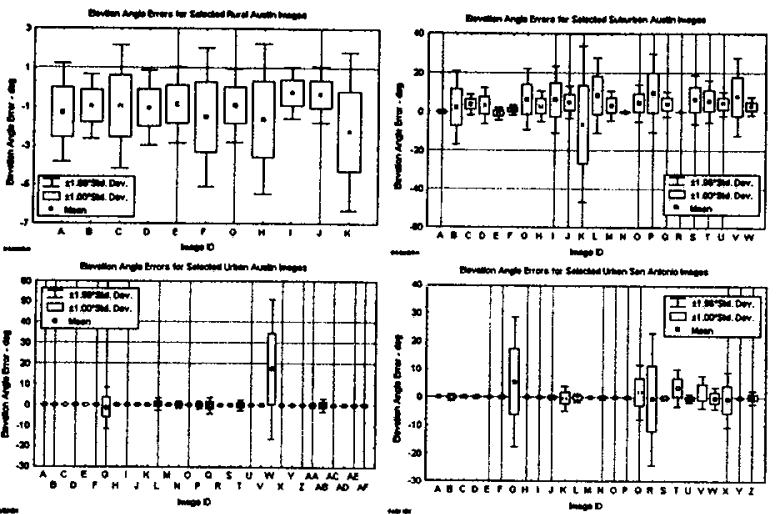

Fig. 10 Elevation errors by image

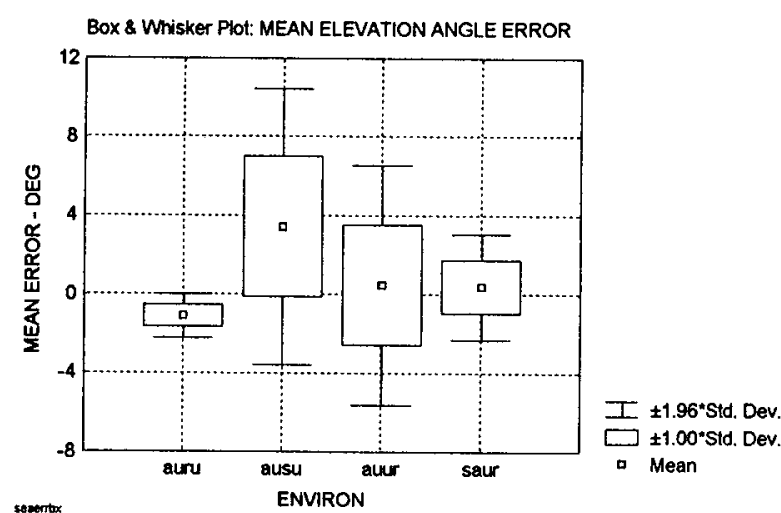

Fig. 11 Mean elevation angle error

The error is nearly normally distributed for the rural and suburban environments, but not for the urban ones.

\section{FUTURE EFFORTS}

Efforts are now under way to derive a full three-state description of images. This will allow the connection of image data to propagation statistics for simulation and prediction purposes.

The programming is being done in $\mathrm{C}$ on a 486-33 platform. Most of the effort so far has been directed towards developing reliable algorithms as opposed to achieving processing speed. The analysis in this report took $120 \mathrm{~s} /$ image. Recompiling the program as written to run on a Sun workstation and a Cray supercomputer resulted in time reductions to 7.2 and $13.5 \mathrm{~s} / \mathrm{image}$, respectively. This is not yet fast enough to analyze video at a 30 images/s rate, but speed optimization has yet to be implemented.

\section{CONCLUSION}

Photogrammetry will provide a powerful tool to derive service predictions for personal and mobile satellite communications systems in any environment. Initial results, although restricted to a binary description of sky visibility, are encouraging and should be of immediate use to satellite system designers. However, more work needs to be done to derive a ternary description for clear, shadowed and blocked states. This will allow one to combine satellite orbit design with propagation statistics to predict performance such as fade probability or diversity gain in a specified environment.

\section{ACKNOWLEDGMENTS}

This effort was supported jointly by Loral Aerospace Corporation, Motorola Inc., and the NASA Propagation Program.

\section{BIBLIOGRAPHY}

[1] Goldhirsh, J. and W. J. Vogel, "Propagation Effects for Land Mobile Satellite Systems: Overview of Experimental and Modeling Results," NASA Reference Publication 1274, February 1992

[2] Vogel, W. J. and U. S. Hong, "Measurement and Modeling of Land Mobile Satellite propagation at UHF and L-Band," IEEE Trans. Ant. and Prop., Vol. 36, No. 5, May 1988

[3] Bovik, A. C., "Digital Image Processing," The University of Texas at Austin, Austin, Texas, 1991

[4] Birdwell, B. N., "Design of an optical sensor system to replicate the effects of signal blockage and shadowing on satellite signal propagation," M.S. Thesis, The University of Texas at Austin, Austin, Texas, May 1993

[5] Rosenfeld, A. and A. C. Kak, Digital Picture Processing, Academic Press, Inc., Orlando, Florida, 1982

[6] Bichsel, M. and P. Seitz, "Object recognition with optimum neural networks," in Neural Networks, Lisboa, P. G. J., Ed., Chapman \& Hall, London, 1992 